\title{
Survey aims to capture patient experience
}

$\mathrm{P}$ atients who have spent time in hospital in Canada will soon be asked to rate their experience as part of an effort by the Canadian Institute of Health Information (CIHI) to document and improve patient care across Canada.

"CIHI has been really focused on health system performance reporting, and the kinds of things you need to measure in order to understand [it]," says Kira Leeb, CIHI's director of health system performance. "Part of that is understanding the patient experience."

Many health systems in Canada have been tracking patient experiences for years, but using myriad systems. In 2012, a group of health system administrators asked CIHI to develop a standardized pan-Canadian survey that would allow them to rate their performance against national benchmarks.

"Comparability is quite important when talking about performance," says Leeb. "You need an objective way of measuring patients' experiences."

CIHI has developed a 49-question survey to evaluate all aspects of a patient's experience in hospital, from their treatment by nurses and doctors, to the hospital environment, to how well their transition through the hospital was managed. Some of the questions were drawn from the US-based Hospital Consumer Assessment of Healthcare Providers and Systems (HCAHPS) survey, with others reflecting aspects of care unique to Canada. The survey will be sent out to, or carried out by phone with, a representative sample of patients within a few weeks of their discharge from hospital. Their anonymized responses will feed back into a central CIHI database, allowing it to analyze the data at a population level.

The survey will help hospitals identify aspects of their patients' experiences that require more work. If the

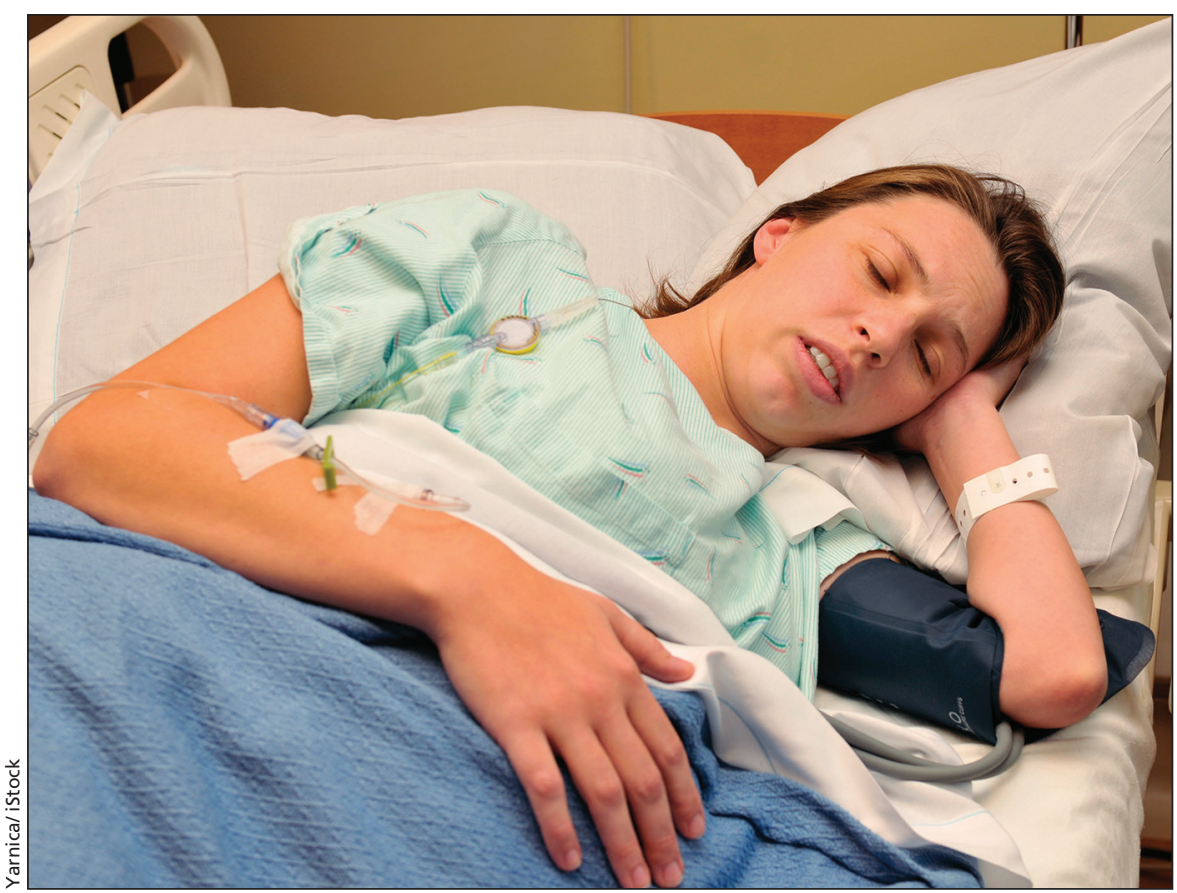

The Canadian Institute of Health Information is launching a pan-Canadian survey of patient care.

results show a systemic problem with particular areas, such as discharges, they may also influence policy changes on a provincial or regional level. In addition, they can help identify how health outcomes and patient experiences relate to one another.

"On an individual level, a patient could have a technically excellent health outcome but a poor hospital experience, or vice-versa," says Leeb. "It's helpful for hospitals to understand where that disconnect occurred."

$\mathrm{CIHI}$ is finishing work on the database and expects to begin collecting information from across Canada later this fall.

Some health systems that took part in the pilot of the survey say they like it. Alberta Health Services (AHS), the first provincial health system to get involved, has been collecting data with the CIHI survey for about a year. Brandi McCormack, director of outcome measurement at AHS, says the health system has been collecting similar data provincially for sometime, using HCAHPS, but she finds it more useful to have a survey that is particular to Canada.

The survey provides AHS with data down to the level of individual hospital units, and is being used for quality assurance, to improve problem areas and to inform care. "It's helpful for our focus on patient-oriented care to have patient experience data," says McCormack.

The data is available to anyone in AHS, and McCormack says use of the information is just starting to ramp up, now that there is a full year's worth available; more people are requesting access and help with interpreting the results.

"I think exciting things are coming with this data," says McCormack. "It's becoming more and more recognized how important the patient voice is." — Brian Owens, St. Stephen, NB

CMAJ 2015. DOI:10.1503/cmaj.109-5172 